\title{
Hybrid Micro-Ring Resonator Hydrogen Sensor Based on Intensity Detection
}

\author{
Kenan Çiçek \\ Department of Electrical and Electronic Engineering, Iğdır University School of Engineering, Iğdır, Turkey \\ Department of Electrical and Electronic Engineering, University of Bristol School of Engineering, Bristol, UK
}

Cite this article as: K. Çiçek, "Hybrid Micro-Ring Resonator Hydrogen Sensor Based on Intensity Detection", Electrica, vol. 18 , no: 2, pp. 167-171, 2018.

\begin{abstract}
In this paper, a photonic integrated device fabricated on a silicon-on-insulator (SOI) platform is studied numerically to investigate its hydrogen sensing potential based on intensity variations. A single-slot hybrid structure consisting of a coaxial micro-ring resonator (MRR) and a palladium (Pd) disk is utilized for this purpose. The results of the numerical study reveal a hydrogen sensing ability of $2.83 \times 10^{-4} /\left(\mathrm{V} / \mathrm{V}-\%\right.$ hydrogen) and limit of detection (LOD) of $9.93 \times 10^{-3}$ which is more than 10 times of that of the hydrogen sensors based on the traditional resonance shift. The proposed hydrogen sensing technique presents a compatible SOl-based technology and also provides a reliable detection of the slightest changes from the zero concentration in an analytical procedure.
\end{abstract}

Keywords: Hydrogen sensor, micro-ring resonator, optic sensor, intensity-based sensor.

\section{Corresponding Author: \\ Kenan Çiçek}

\section{E-mail:}

eexkc@my.bristol.ac.uk

Received: 21.11 .2017

Accepted: 15.05 .2018

C Copyright 2018 by Electrica

Available online at

http://electrica.istanbul.edu.tr

DOI: 10.26650/electrica.2018.56722

\section{Introduction}

Hydrogen is the most common element, making up $75 \%$ of the mass of the entire universe and has been used as a fuel for many decades in a wide range of applications. Hydrogen requires a high sensitive detection for human safety not only because it possesses a wide flammability range of an explosive nature (lower and upper explosive limits of $4 \%$ and $75 \%$ in air, respectively) but also it's great leaking tendency feature [1]. Many studies have been carried out for the purpose of hydrogen detection including fiber-based sensors, chemical sensors, photonic crystal sensors and micro-cavity optical sensors [2-5]. Among these, the micro-cavity/micro-resonator sensors have high potential in high sensitive detection [6, 7]. Hybrid structures of micro-resonators which might in hold different materials such as Pd is considered as a high-sensitive, low-cost, compact, durable, and high performance hydrogen detector compared to conventional hydrogen gas detecting instruments $[5,8]$.

Typical Pd involved optic micro-ring resonator (MRR) hydrogen sensor utilizes resonance-shift analysis to probe the presence of hydrogen in the sensing chamber. In such a sensing scheme the resonance wavelength/frequency alters with the change in hydrogen concentration of the sensor system. In order to detect low concentration levels, a generally high quality factor is required for a detectable resonance-shift [9]. It is well-known that due to the absorption feature of metals, the quality factor of a metal involved optical sensing system is typically low, which consequently leads to a lower detection limit $[10,11]$. In addition, the perceptibility of the resonance-shift of this kind of optical sensor becomes smaller as the bandwidth of the resonance decreases, which also results in a lower detection limit [12].

As an alternative, intensity-based optical sensors may be advantageous over those based on resonance-shift. The intensity-based sensors measure the output intensity variation of a resonance peak such that the absorption due to the metal component helps to increase the performance rather than to decrease. In addition, such sensors are free from bandwidth restrictions so that they can operate in a wide-range of wavelength. 
It has been reported that a single-slot hybrid MRR hydrogen sensor provides a sensitivity 23 times higher than its counterparts [8]. However, this resonance-shift based sensor has quality-factor issues due to $\mathrm{Pd}$ presence which is vital for a higher detection limit as mentioned before. To solve this problem, intensity-based analysis can be performed considering advantageous over resonance-shift that is mentioned above. Therefore, in this paper, the single-slot hybrid MRR hydrogen sensor is re-analysed based on intensity changes in case of hydrogen entrance to the sensing chamber and results are discussed.

\section{Hybrid Micro-Ring Resonator Sensor}

\section{Proposed Geometry}

The proposed hydrogen sensor geometry is presented in Figure 1. As it can be seen from Figure 1-a, the structure consists of an SOI (Silicon on Insulator) based micro-ring resonator enclosing a Pd disk with a gap distance of $w_{\text {slot }}\left(w_{\text {slot }}=R_{\text {in }}-R_{P d}\right)$. The 4-port MRR with an outer radius of $R_{\text {out }}$ and a width of $W_{M R R}$ $\left(W_{M R R}=R_{\text {out }}-R_{\text {in }}\right)$ couples to a pair of $400 \mathrm{~nm}$ width bus waveguides nearby and generates whispering galley modes (WGM) within an air sensing medium. In the calculations, the refractive indices of air and silicon MRRs are used as 1.000293 and 3.476, respectively for Telecom wavelengths. The optical parameters of $\mathrm{Pd}$ is provided from the Reference [13].

Intensity distribution of excited WGMs in MRR is highly depended on $W_{M R R}$. As can be seen from Figure $1 \mathrm{~b}, \mathrm{c}$ in a single mode waveguide with $W_{M R R}=300 \mathrm{~nm}$ and $W_{M R R}=400 \mathrm{~nm}$, respectively, TE polarised e- field forms different distribution patterns. The figures show that the light tightly confines in a wider waveguide than in a narrower one. In addition, the evanescent field at the wider waveguide fades away within a short distance which is highly effective on sensing performance of the sensor under investigation of this paper. Therefore, in this work, sensing performance is numerically investigated based on the geometries which include two sizes of MRR waveguide width of $300 \mathrm{~nm}$ and $400 \mathrm{~nm}$.

This study is carried out using two-dimensional Finite Difference Time Domain (FDTD-Meep) method to simulate the proposed 4-port MRR structure to investigate its hydrogen sensing potential. The computer based simulations are performed in $2 \mathrm{D}$ because of the limitation of resources. Even if 3D simulations are needed for real-world applications, 2D calculations allow you to see 3D performance with approximate numerical results with less resource and time consumption [14]. A grid size as small as $2 \mathrm{~nm}$ and sufficient working time is used for high accuracy and reliability of simulations. For calculations, we assume that the silicon MRR and the combined waveguide system are in the air sensing environment.

\section{Operation Principle}

An incident beam with Gaussian spectrum is injected into the system via input port as sketched in Figure 1 a. Excited WGMs

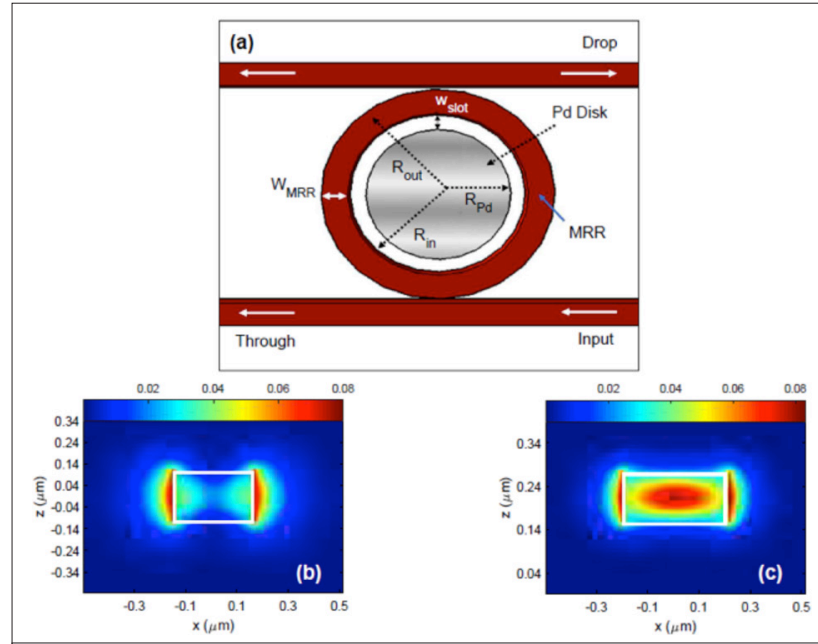

Figure 1. Schematic architecture of proposed hydrogen sensing device (a); e- field distribution at the cross-section of $W_{M R R}=300$ $\mathrm{nm}(\mathrm{b})$; and $\mathrm{W}_{\text {MRR }}=400 \mathrm{~nm}$ waveguide at the wavelengths of 1512 $\mathrm{nm}$ and $1517 \mathrm{~nm}$ (c), respectively

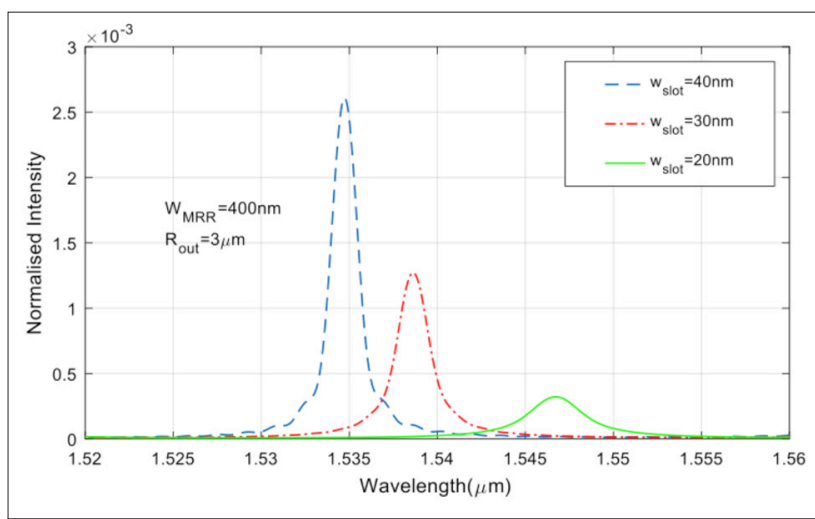

Figure 2. Resonant wavelength changes in case of $\mathrm{w}_{\text {slot }}=40 / 30 / 20$ $\mathrm{nm}$ observed from a $M_{R R}$ sensor with $w_{\text {slot }}=400 \mathrm{~nm}$ and $R_{\text {out }}=3 \mu \mathrm{m}$.

within the resonator, interact with the surrounding medium via evanescent waves, the tail of WGMs which lie along the ring cross-section, Figure 1 b, c. It is well-known that any change in sensors cladding or surrounding medium causes WGMs to shift in wavelength/frequency spectrum.

With the introduction of hydrogen to the sensing chamber, the resonance wavelength experiences a redshift as shown in figure 2. In classical perspective of sensing, this shift is directly correlated with hydrogen concentration which has already been studied [8]. It also can be seen from the figure that along with the wavelength shift there is a significant change in intensity of resonance during the sensing process. This is mainly because of the existence of $\mathrm{Pd}$ which as a metal possesses a complex refractive index coefficient at a wide range of wavelength causing considerable absorption. Thus, a constant flow of hydrogen into the sensing chamber causes the Pd disk to expand in diameter leading to contact more with evanescent 


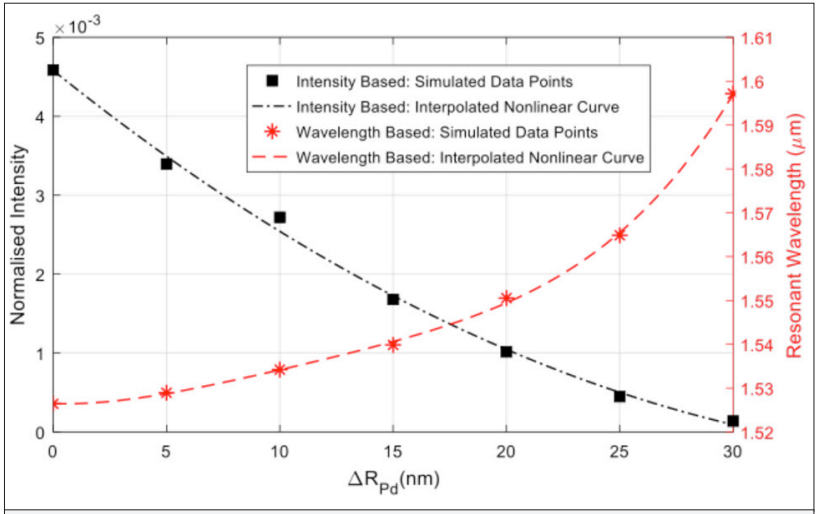

Figure 3. Interpolation of intensity (left hand side $y$-axis)/wavelength (right hand side $y$-axis) based simulated data for a geometry where $R_{\text {out }}=1 \mu \mathrm{m}, R_{\text {Pd }}=0.66 \mu \mathrm{m}, W_{\text {MRR }}=300 \mathrm{~nm}$ and $w_{\text {slot }}=30$ $\mathrm{nm}$. For these geometry values $5 \mathrm{~nm} \mathrm{R}_{\mathrm{Pd}}$ intervals $\left(\Delta \mathrm{R}_{\mathrm{Pd}}\right)$ corresponds to $C_{\text {hyd }}=8.57 \%$ assuming linear volumetric expansion of $\mathrm{Pd}$ with $\mathrm{H}_{2}$

field and resulting an exponential decrease in intensity of the WGMs as shown in Figure 3.

It is clear from the figure that the slope of intensity plot decreases as $w_{\text {slot }}$ decreases $\left(R_{P d}\right.$ increases). This means that the rate of intensity change exponentially decreases as the Pd disk approaching the MRRs inner face. This phenomenon occurs in resonance-shift based sensors in such a way that any change in $\mathrm{w}_{\text {slot }}$ emerges an inversely proportional effect on resonance wavelength shift, as shown in Figure 3.

In the following section, the hydrogen sensing potential of intensity-based hybrid MMR sensor is investigated for a hydrogen concentration range of $0 \%$ to $1 \%$, a range that allows a reversible expansion in $\mathrm{Pd}$.

\section{Results}

It is a fact that, palladium lattice expends $0.087 \%$ within $1 \%$ $\mathrm{H}_{2}$ sensing environment [15]. In the reference of this, numerical calculations are performed for various geometry parameters of sensor. Each sensor geometry is simulated changing the $\mathrm{Pd}$ radius for each calculation loop assuming the change is due to the hydrogen absorption. However, the $2 \mathrm{~nm}$ grid size employed in this study is not sufficient for pico-meter level changes. Therefore, the simulations are performed changing the $\mathrm{Pd}$ size with $5 \mathrm{~nm}$ intervals at each calculation loop. Then, resulted $\Delta \mathrm{I}$ (intensity change) values are interpolated by non-linear curve fitting to observe at the pico-meter level corresponding to small hydrogen concentration changes as presented in Figure 3. Under all this assumption, the relation between hydrogen concentration $\left(C_{\text {hyd }}\right)$, in volume/volume percent ( $\left.v / v-\%\right)$, and $\Delta \mathrm{l}$ is investigated.

Figure 4 shows $\Delta \mathrm{I}$ values as a function of $C_{\text {hyd }}$ observed from drop port of the sensors for a range of geometric parameters.
From the figure, it is seen that, both $\mathrm{w}_{\text {slot }}$ and $\mathrm{W}_{\text {MRR }}$ have a large effect on $\Delta \mathrm{l}$. Figure $4 \mathrm{a}$, shows that, when a $300 \mathrm{~nm}$ wide MRR with $R_{\text {out }}=1 \mu \mathrm{m}$ and $\mathrm{w}_{\text {slot }}=40 \mathrm{~nm}$ is employed, $\Delta$ l gradually increases up to $1.4 \times 10^{-4}$ along with the hydrogen concentration increment in the range of $C_{\text {hyd }}=0 \%$ to $C_{\text {hyd }}=1 \%$. This value reaches to $1.01 \times 10^{-4}$ and $0.7 \times 10^{-4}$ with smaller slot sizes of $\mathrm{w}_{\text {slot }}=30 \mathrm{~nm}$ and $\mathrm{w}_{\text {slot }}=20 \mathrm{~nm}$, respectively. While $R_{\text {out }}$ increases from $1 \mu \mathrm{m}$ to $3 \mu \mathrm{m}$ the rate of $\Delta \mathrm{l}$ increases directly with the $\mathrm{w}_{\text {slot }}$ size. As presented in Figures $4 \mathrm{~b}, \mathrm{c} ; \Delta \mathrm{l}$ reaches higher values of $1.8 \times 10^{-4}$ and $2.2 \times 10^{-4}$ for devices with $R_{\text {out }}=2 \mu \mathrm{m}$ and $\mathrm{R}_{\text {out }}=3 \mu \mathrm{m}$, respectively when $w_{\text {slot }}=40 \mathrm{~nm}$. It is evidence from the Figures $4 \mathrm{a}-\mathrm{c}$, that regardless of $\mathrm{R}_{\text {out }}$ the highest $\Delta \mathrm{l}$ rates are observed when $\mathrm{w}_{\text {slot }}=40 \mathrm{~nm}$. On the other hand, the rate of $\Delta \mathrm{l}$ in response to hydrogen concentration slightly increases for $W_{M R R}=400 \mathrm{~nm}$ wide MRRs as compared to $W_{M R R}=300 \mathrm{~nm}$, as shown in Figure $4 \mathrm{e}, \mathrm{f}$. As such, the highest shift can be obtained for a design where $\mathrm{w}_{\text {slot }}=40 \mathrm{~nm}$ and $\mathrm{R}_{\text {out }}=3 \mu \mathrm{m}$ is around $2.8 \times 10^{-4}$. It needs to be noted that, in Figure $4 \mathrm{~d}$ the observed $\Delta \mathrm{l}$ for the geometry with $\mathrm{w}_{\text {slot }}=20 \mathrm{~nm}$ and $\mathrm{R}_{\text {out }}=1 \mu \mathrm{m}$ is higher than the one with $\mathrm{w}_{\text {slot }}=40 \mathrm{~nm}$ for the same radius.

This is due to the decreasing behaviour of the slope of the intensity plot for this particular geometry through the $\mathrm{w}_{\text {slot }}$ other computed geometries (presented in other sub-figures) exhibit an increasing trend for the same plot. The reason for this intensity trend difference might be because of the loss (includes scattering, bending and absorption losses) rate in case of each $\mathrm{w}_{\text {slot }}$ value [6].

In order to determine the efficiency of the sensor, the sensitivity $\left(S=\Delta \mathrm{l} / \Delta \mathrm{C}_{\text {hyd }}\right)$ as function of hydrogen concentration is calculated. According to the calculations, hydrogen could be detected with a sensitivity of as high as $2.20 \times 10^{-4} /(\mathrm{v} / \mathrm{v}-\%$ hydrogen) employing an $\mathrm{R}_{\text {out }}=3 \mu \mathrm{m}$ MRR system where $\mathrm{w}_{\text {slot }}=40$ $\mathrm{nm}$ and $\mathrm{W}_{\text {MRR }}=300 \mathrm{~nm}$. In addition, $S$ could be further increased up to $2.83 \times 10^{-4} /\left(\mathrm{v} / \mathrm{v}\right.$ - \% hydrogen) with same $\mathrm{R}_{\text {out }}$ and $\mathrm{w}_{\text {slot }}$ but a wider resonator width of $W_{M R R}=400 \mathrm{~nm}$.

Another factor to describe the performance of an optical sensor is the Limit of detection (LOD) which is the lowest concentration of hydrogen that can be distinguished from the zero concentration level. LOD of proposed intensity-based sensor is calculated based on the standard deviation of the response $\left(\mathrm{L}_{\mathrm{r}}\right)$ of each linear curve and the slope of the calibration curve $\left(\mathrm{L}_{\mathrm{sl}}\right)$ of $\Delta \mathrm{I}$ vs $\mathrm{C}_{\text {hyd }}$ at levels approximating the LOD according to the formula $L O D=\left(3.3 \times L_{r}\right) / L_{s \mid}[16] . L_{r}$ is determined based on the standard deviation of $y$-intercepts of regression lines and both $L_{r}$ and $L_{s l}$ are computed via MS Excel. It is found that the intensity-based hybrid MMR sensor offers an LOD of $9.93 \times 10^{-3}$ for a geometry that present highest sensitivity. In comparison with this study, the LOD of the most sensitive geometry in reference [8] is also calculated with the same theory and found to be $7.16 \times 10^{-2}$ which is around 10 times higher than the one with intensity- based sensing. 


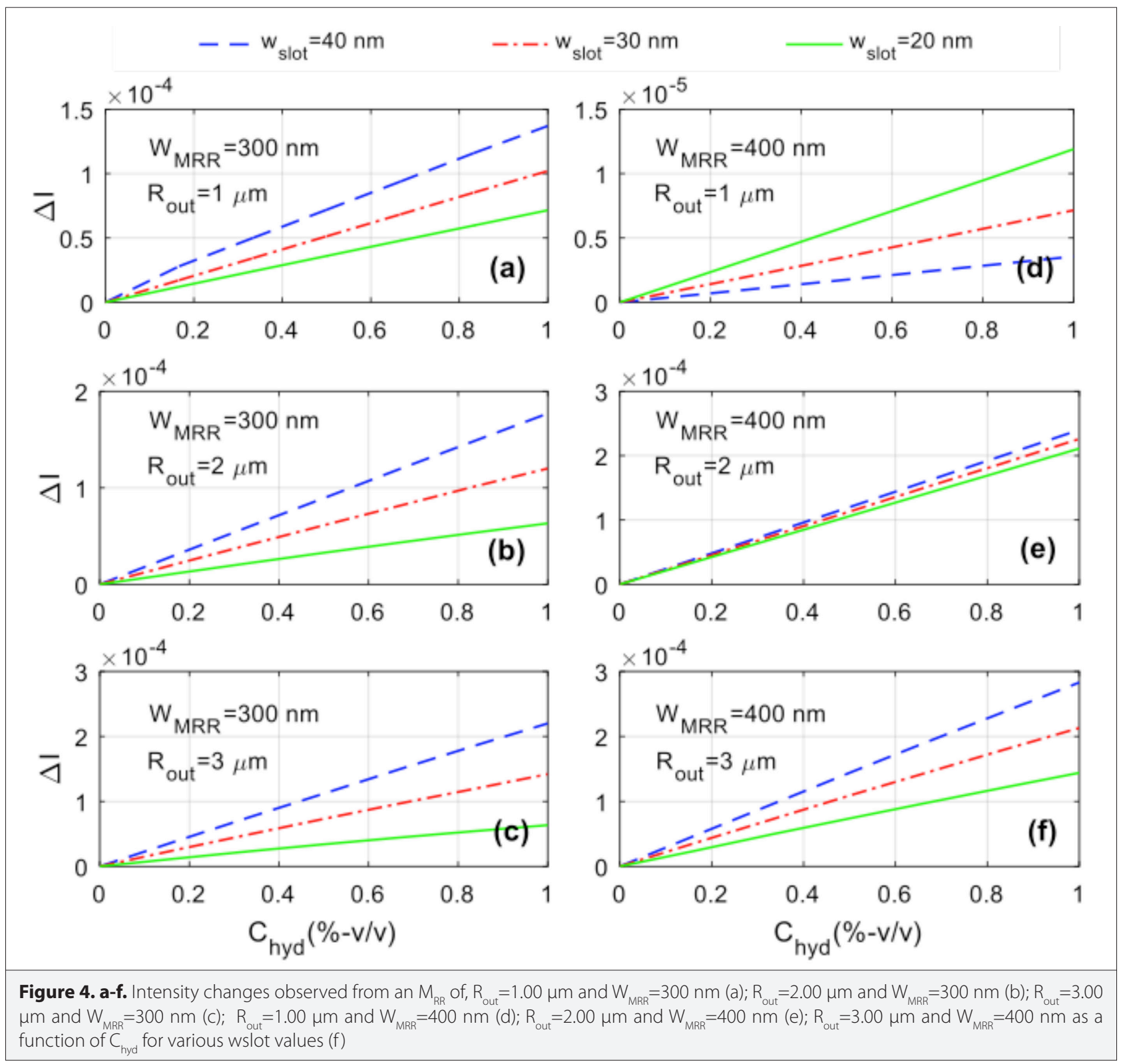

\section{Conclusion}

This paper presents a numerical study of a hybrid MRR system for hydrogen sensing purposes based on intensity change. The sensitivity of $2.83 \times 10^{-4} / \%$ hydrogen and the LOD of $9.93 \times 10^{-3}$ testify the high performance of intensity-based hybrid sensor in hydrogen gas sensing. Thus, the suggested novel technique is highly promising with its sensitive, highly reliable, compact, robust, low cost and small footprint hydrogen detection ability.

Peer-review: Externally peer-reviewed.

Conflict of Interest: The authors have no conflicts of interest to declare.

Financial Disclosure: The authors declared that this study has received no financial support.

\section{References}

1. H. Gu, Z. Wang,Y. Hu, "Hydrogen Gas Sensors Based on Semiconductor Oxide Nanostructures", Sensors, vol. 12, no. 5, 5517-5550, 2012.

2. R. Tabassum, S.K. Mishra, B.D. Gupta, "Surface plasmon resonance based ber optic hydrogen sulphide gas sensor utilizing $\mathrm{Cu} / \mathrm{ZnO}$ thin Ims", Phys Chem Chem Phys, vol. 15, no. 28, 11868-11874, 2013.

3. S. Kim, J. Choi, M. Jung, S. Joo S, S. Kim, "Silicon carbide-based hydrogen gas sensors for high-temperature applications", Sensors, vol. 13, no. 10, 13575-13583, 2013.

4]. V.P. Minkovich, D. Monzon-Hernondez, J. Villatoro, G. Badenes, "Microstructured optical ber coated with thin Ims for gas and chemical sensing", Optics Express, vol. 14, 8413-8418, 2006.

5. M. Eryurek, Y. Karadağ, N. Taşaltın, N. Kılınç, A. Kiraz, "Optical sensor for hydrogen gas based on a palladium-coated polymer mi- 
croresonator" Sensors and Actuators B: Chemical, vol. 212, 78-83, 2015.

6. L. Zhou, X. Sun, X. Li, J. Chen, "Miniature Microring Resonator Sensor Based on a Hybrid Plasmonic Waveguide", Sensors, vol. 11, no. 7, 6856-6867, 2011.

7. A. Daraei, M. E. Daraei, "Thin cylindrical slot in an optical microdisk cavity for sensing biomaterials", Appl Phys A, vol. 123, 216-222, 2017.

8. K. Cicek, M. Eryrek, A. Kiraz, "Single-slot hybrid microring resonator hydrogen sensor" J. Opt. Soc. Am. B, VOL. 34, NO. 7, 1465-1470, 2017.

9. P.S. Pedersen, P. S. Nunes, S. Xiao, N.A. Mortensen, "Material Limitations on the Detection Limit in Refractometry" Sensors, vol. 9, 8382-8390, 2009.

10. L. Huang, H. Yan, X. Xu, S Chakravarty, N Tang, H Tian, R. T. Chen, "Improving the detection limit for on-chip photonic sensors based on subwavelength grating racetrack resonators" Optics Express, vol. 25, 10527-10535, 2017.
11. Y. Liu, W. Zhou, Y. Sun, "Optical Refractive Index Sensing Based on High-Q Bound States in the Continuum in Free-Space Coupled Photonic Crystal Slabs", Sensors, vol. 17, 1861-1873, 2017.

12. Z. Xia, Y. Chen, Z. Zhou, "Dual Waveguide Coupled Microring Resonator Sensor Based on Intensity Detection", IEEE J Quantum Elect, vol. 44, 100-107, 2008.

13. A. D. Rakic, A B. Djurisic, J. M. Elazar, M. L. Majewskii, “Optical properties of metallic Ims for vertical-cavity optoelectronic devices", Appl Opt, vol. 37, 5271-5283, 1998.

14. M. Gabalis, D. Urbonas, R. Petruskevicius, "A perforated microring resonator for optical sensing applications", Journal of Optics, vol. 2016, 105003-105009, 2017.

15. J. S. Noh, J. M. Lee, W. Lee, "Low-Dimensional Palladium Nanostructures for Fast and Reliable Hydrogen Gas Detection" Sensors, vol. 11, 825-851, 2011.

16. A. Shrivastava, V. Gupta, "Methods for the determination of limit of detection and limit of quantitation of the analytical methods" Chronicles of Young Scientists, vol. 2, 21-25, 2011.

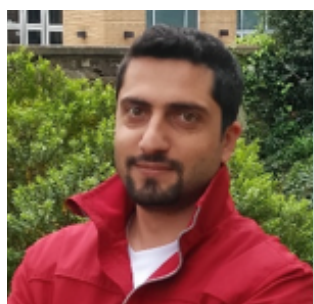

Kenan Çicek, I have been graduated from University of Bristol with a PhD degree in 2016. Currently I am an assistant professor in Iğdır University, Turkey. My specialty is integrated optics and photonic devices. 